\title{
On the origin of X-shaped radio-sources: New insights from the properties of their host galaxies ${ }^{\star}$
}

\author{
A. Capetti ${ }^{1}$, S. Zamfir ${ }^{1,2}$, P. Rossi ${ }^{1}$, G. Bodo ${ }^{1}$, C. Zanni ${ }^{3}$, and S. Massaglia ${ }^{3}$ \\ ${ }^{1}$ Istituto Nazionale di Astrofisica (INAF) - Osservatorio Astronomico di Torino, Strada Osservatorio 20, 10025 Pino Torinese, \\ Italy \\ ${ }^{2}$ Universitatea din Bucuresti, Facultatea de fizica atomica si nucleara, Bucuresti-Magurele, PO-BOX MG-11, Romania \\ 3 Istituto di Fisica Generale, Universitá di Torino, Via Giuria 1, 10125 Torino, Italy
}

Received 7 May 2002 / Accepted 19 July 2002

\begin{abstract}
A significant fraction of extended radio sources presents a peculiar X-shaped radio morphology: in addition to the classical double lobed structure, radio emission is also observed along a second axis of symmetry in the form of diffuse wings or tails. We re-examine the origin of these extensions relating the radio morphology to the properties of their host galaxies. The orientation of the wings shows a striking connection with the structure of the host galaxy as they are preferentially aligned with its minor axis. Furthermore, wings are only observed in galaxies of high projected ellipticity. Hydrodynamical simulations of the radio-source evolution show that X-shaped radio-sources naturally form in this geometrical situation: as a jet propagates in a non-spherical gas distribution, the cocoon surrounding the radio-jets expands laterally at a high rate producing wings of radio emission, in a way that is reminiscent of the twin-exhaust model for radio-sources.
\end{abstract}

Key words. galaxies: active - galaxies: elliptical and lenticular; cD - galaxies: jets

\section{Introduction}

Extended radio sources have been historically classified on the basis of their radio morphology, the main division being based on their edge darkened or edge brightened structure that leads to the identification of the Fanaroff-Riley classes I and II (Fanaroff \& Riley 1974). The characteristic structure of FR II sources is dominated by two hot spots located at the edges of the radio lobes that, in most cases, show bridges of emission linking the core to the hot spots. The presence of significant distortions in the bridges was recognized since early interferometric imaging of 3C sources (see e.g. Leahy \& Williams 1984). Distortion in FR II can be classified in two general classes: mirror symmetric (or C-shaped) when the bridges bend away from the galaxy in the same direction, or centro symmetric when they bend in opposite directions forming an X-shaped or $Z$-shaped radio source, depending on the location of the point of insertion of the wings. In many $\mathrm{X}$-shaped sources the radio emission along the secondary axis, although more diffuse, is still quite well collimated and can be even more extended than the main double lobed structure.

Send offprint requests to: A. Capetti, e-mail: capetti@to. astro.it

* Based on observations obtained at the Italian Telescopio Nazionale Galileo (TNG) operated on the island of La Palma by the Centro Galileo Galilei of the CNAA (Consorzio Nazionale per l'Astronomia e l'Astrofisica) at the Spanish Observatorio del Roque de los Muchachos of the Instituto de Astrofisica de Canarias.
C-shaped morphologies are observed also in FR I radiogalaxies although in these sources the distortions affect their jets rather than their lobes and they give rise to the typical shape of Narrow Angle Tails, where the opposite jets bend dramatically and become almost parallel one to the other. Another common morphology for FR I is that of centrosymmetric S-shaped sources. Conversely, there are no examples of X-shapes among FR I.

There is now a general agreement that the $\mathrm{C}$-shaped radio sources form when they are in motion with respect to the external medium: jets or bridges are bent by the ram pressure of the surrounding gas. Models successfully reproduced the morphology of FR I Narrow Angle Tails (see e.g. O'Dea \& Owen 1986) and the extension of this scenario to FR II bridges appears quite natural.

Concerning the X- or Z-shaped sources several mechanism have been proposed for their origin. Ekers et al. (1978) suggested that the tails of radio emission in one of these sources, NGC 326, are the result of the trail caused by a secular jet precession (see also Rees 1978). A similar model accounts for the morphology of 4C 32.25 (Klein et al. 1995). In a similar line, Wirth et al. (1982) noted that a change in the jet direction can be caused by gravitational interaction with a companion galaxy. Recently Dennett-Thorpe et al. (2002), from the analysis of spectral variations along the lobes, proposed that the jet reorientation occurs over short time scales, a few Myr, and are possibly associated to instabilities in the accretion disk that 
Table 1. Optical and radio PA of the winged radiosources.

\begin{tabular}{lcccc}
\hline \hline Name & Optical PA & Wings PA & Offset & Radio PA \\
\hline 3C 52 & 55 & -65 & 60 & 25 \\
3C 63 & 80 & -45 & 55 & 30 \\
3C 136.1 & -80 & 10 & 90 & -70 \\
3C 192 & -85 & 60 & 55 & -55 \\
3C 223.1 & 40 & -40 & 80 & 15 \\
3C 315 & 35 & -45 & 80 & 10 \\
3C 403 & 35 & -50 & 85 & 85 \\
4C 12.03 & -25 & 70 & 85 & 15 \\
4C 32.25 & 90 & -5 & 85 & 60 \\
\hline
\end{tabular}

cause a rapid change in the jet axis. In all these models, the secondary axis of radio emission represents a relic of the past activity of the radio source.

An alternative interpretation was suggested by Leahy \& Williams (1984) and Worrall et al. (1995). They emphasize the role of the external medium in shaping radio sources, suggesting that buoyancy forces can bend the back-flowing material away from the jet axis into the direction of decreasing external gas pressure.

In this Paper we present a different scenario based on evidence for a strong connection between the properties of the radio emission and those of the host galaxy, a comparison that has been overlooked in the past but that provides crucial new insights on the origin of X-shaped radio-sources. In fact, in Sect. 2 we compare the radio and host galaxy orientation of $\mathrm{X}$-shaped radio sources showing that there is a close alignment between the radio wings and the galaxy minor axis. We also show that X-shaped sources occur exclusively in galaxies of high ellipticity. Numerical simulations, presented in Sect. 3, provide support to the idea that X-shaped radio-sources form naturally in this geometrical situation. Finally, in Sect. 4 we discuss the implications of our results that are summarized in Sect. 5.

\section{Host galaxy properties of X-shaped radio sources}

\subsection{The sample}

We searched in the literature for X-shaped radio sources. $\mathrm{X}$-shaped distortion are quite common in FR II but we prefer to concentrate on the 9 objects in which the secondary radio axis is well developed, with a size of at least $80 \%$ of that of the main radio axis. We therefore excluded objects (like 3C 274.1, 3C 341 or 3C 381, see Leahy \& Williams 1984) in which the radio wings are present but are considerably smaller. The selected sources are listed in Table 1 where we present also the relevant parameters of the radio source and of the host galaxy.

The properties of the host galaxies have been derived from the analysis of infrared $K$ band images obtained at the Telescopio Nazionale Galileo in July 2000 as part of a complete infrared survey of $3 \mathrm{C}$ radio-galaxies with redshift $z<0.3$ (see Capetti et al. 2002 for details on these observations). The data also provide us with a control sample of 18 radio-galaxies (with the further observational constraint of being located in the sky at $15 \mathrm{~h}<\mathrm{RA}<02 \mathrm{~h}$ ).

\subsection{Host galaxy and radio wings orientation}

For each source we determined the orientation of the radio wings by connecting the outer edges of the detected radio emission. To determine the galaxy position angle we used the TNG K band images. For the two winged radio sources not belonging to the $3 \mathrm{C}$ sample we instead used optical images from the Digitized Sky Survey. From ellipse fitting of each galaxy we determined the behaviour of both the position angle and ellipticity of the isophotes. Typical errors in the PA determination are estimated to be $\sim 5^{\circ}$. The values of radio and host galaxy position angles derived for each source are given in Table 1 and a histogram of their difference is shown in Fig. 2. Clearly, the PA difference avoids values smaller than $\triangle \mathrm{PA}<55^{\circ}$ and shows a strong concentration at high values with an average value of $75^{\circ}$. With a Kolmogorov-Smirnov test we estimated that the observed distribution has only a probability of $5 \%$ to be drawn from a random (uniform) distribution.

Radio wings therefore appear to be oriented along a direction that is almost perpendicular to the galaxy major axis and consequently along its minor axis (see Fig. 1 for two representative examples). The connection between radio and galaxy axis becomes stronger considering the effects of projection. In fact, while for a highly flattened galaxy the major axis is always orthogonal to the perpendicular to the galaxy disk, for a more complicated (and realistic) galaxy shape, such as a triaxal ellipsoid, projection can only decrease the angle between the two axis and populate also relatively lower values of $\triangle \mathrm{PA}$, similarly to what is observed in our distribution. We then conclude that the orientation of the radio wings and galaxy major axis is closely connected and, considering the effects of projection, they are plausibly intrinsically perpendicular one with respect to the other.

\subsection{Radio morphology and host ellipticity}

A second result obtained from the analysis of the host galaxy images is that winged radio sources appear to be associated to galaxies of high ellipticity isophotes. To support quantitatively this visual impression in Fig. 3 we compare the distribution of ellipticity of the seven winged source from the $3 \mathrm{C}$ (the DSS images of 4C 12.03 and 4C 32.25 do not allow us an accurate determination of ellipticity) with the control sample. The ellipticity has been measured at a fixed distance ( $\left.3^{\prime \prime}\right)$ from the galaxy center. Clearly, the winged sources are only found for high values of ellipticity. A Student test indicates that the probability that the ellipticity distribution of winged sources and the control sample are drawn from the same population is only $2.8 \%$.

\section{Numerical simulations}

In the previous section we showed the existence of a connection between the host galaxy and radio properties: X-shaped radio 
3C 223.1

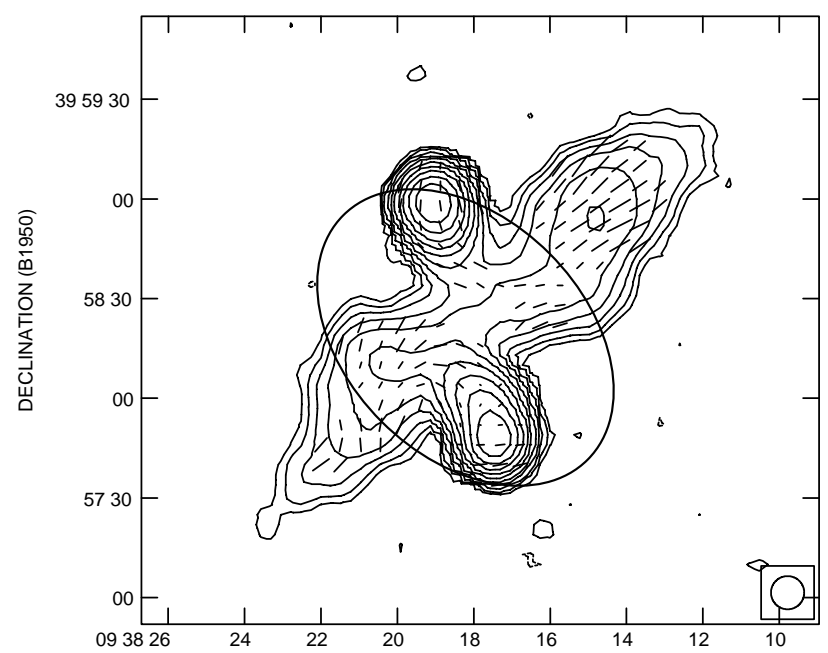

3C 403

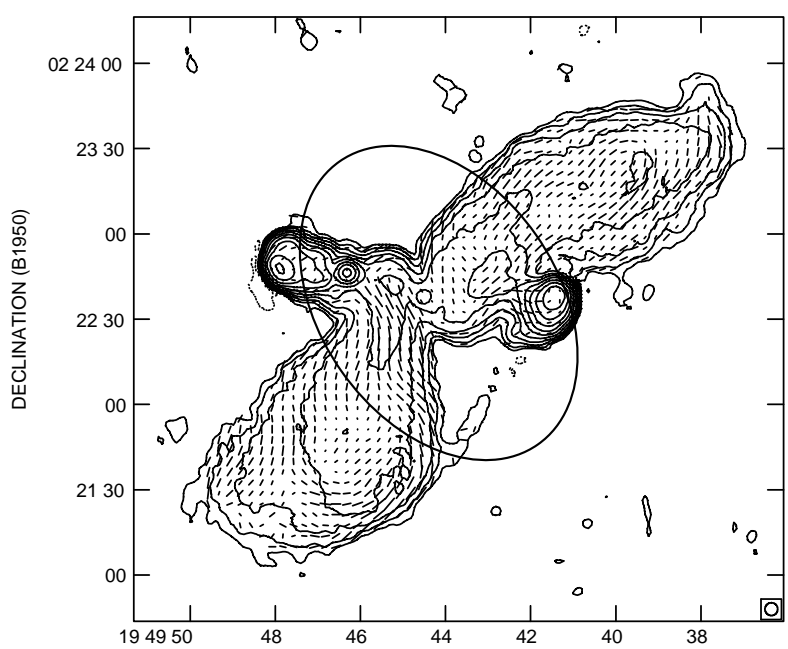

Fig. 1. Superposition of the host galaxy shape (not in scale) onto the radio maps (taken from Dennett-Thorpe et al. 2002, MNRAS, 330, 609 (Blackwell Publishing)) for two X-shaped radio-sources.

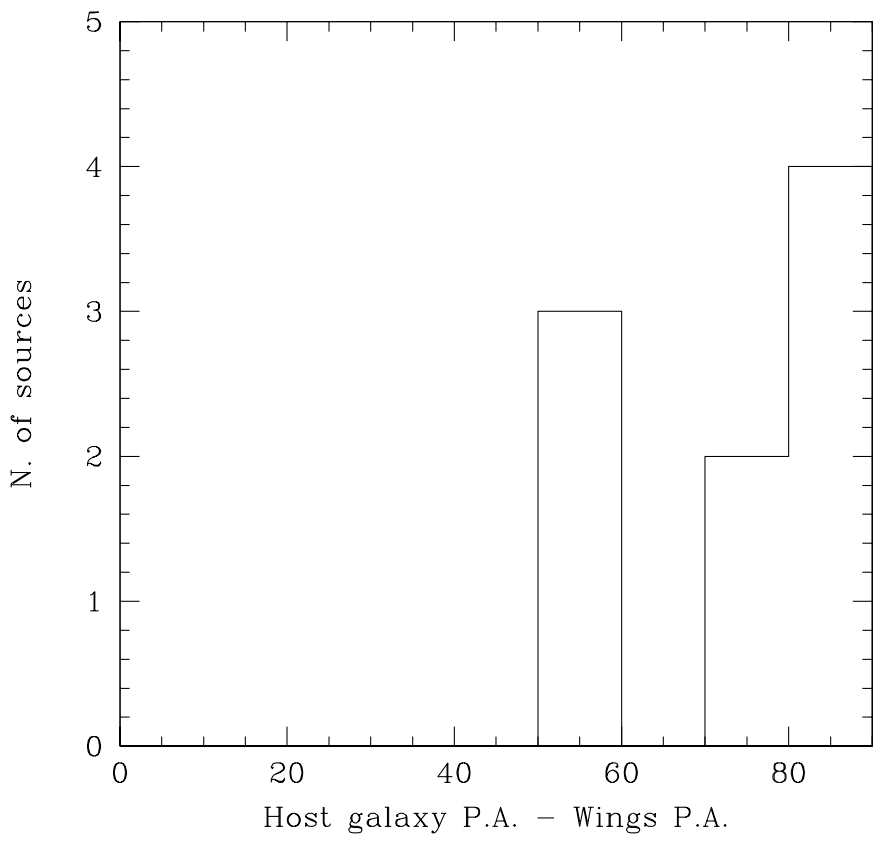

Fig. 2. Relative orientation of the major axis of the host galaxy and of the secondary axis of radio emission.

sources have their wings aligned with the galaxy minor axis and wings are present only in galaxies of high ellipticity. These results suggest that the shape of the galaxy potential, which determines the distribution of external gas, plays an important role in the evolution of the radio-source. To further investigate this issue we performed numerical simulations of the propagation of a jet in a stratified medium with an elliptical symmetry. Our aim is to show that an effect exists and that it can originate morphologies analogous to the observed $\mathrm{X}$-shaped radio sources. Therefore we did not perform an extensive parameter study but we considered two exemplificative cases, with the jet oriented along and perpendicularly to the galaxy's major axis.

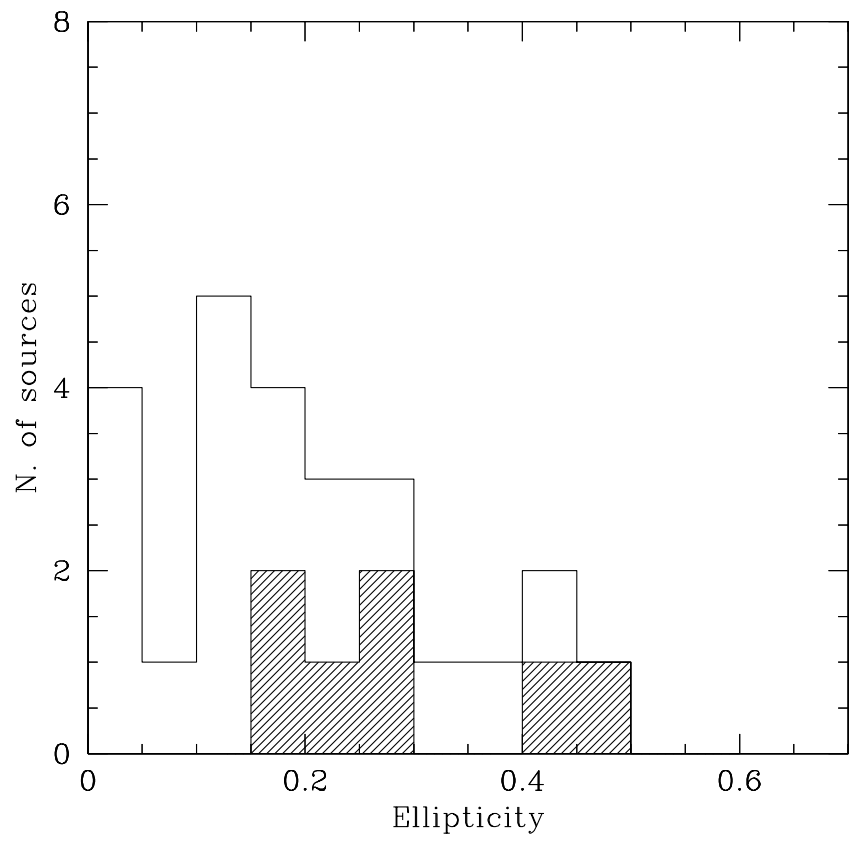

Fig. 3. Host galaxy ellipticity of the X-shaped radio-sources (shaded histogram) compared to a reference sample of 3C FR II radio-galaxies.

The adopted external gas distribution has an ellipsoidal shape and, in Fig. 4, we represent the density profiles along the major and minor axis. The width $r_{\mathrm{c}}$ of the central plateau along the major axis is chosen as our unit of length. The gas is isothermal and the density distribution is kept in equilibrium by an appropriated gravitational potential.

The integration is performed in cylindrical geometry, the computational domain extends up to $6 r_{\mathrm{c}}$ in the horizontal direction and up to $9 r_{\mathrm{c}}$ in the vertical direction and is covered by $704 \times 1024$ grid points. We used symmetry boundary conditions at the bottom and left boundaries and free outflow conditions at the top and right boundaries. The basic equations of conservation have been integrated with a two-dimensional version of the Piecewise Parabolic Method (Colella \& Woodward 1984). 


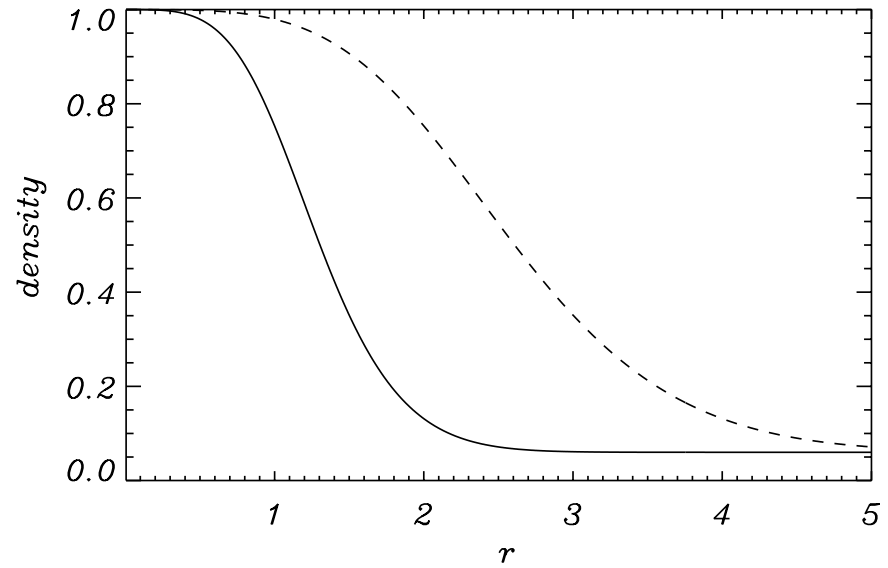

Fig. 4. Equilibrium density profile of the adopted external gas distribution. The gas has an ellipsoidal shape and here we represent the density profiles along the major (dashed line) and minor (solid line) axis.

The jet has a Mach number $M=60$, defined with respect to the sound speed in the ambient medium, and a density $3 \times$ $10^{-3}$ times the central value in the external medium.

In Fig. 5 we show density images representing the evolution of the radio-source in the anisotropically stratified medium. The computation has been performed on one quarter of the panels, but, for clarity, we have reproduced also the symmetric regions. The top panels refer to the case when the jet is oriented parallel to the major axis of the distribution, while the lower panels to the case when the jet is oriented parallel to the minor axis.

In the very initial stages, when the radio source size is smaller than the central density plateau of the external gas, the jet inflates a cocoon whose shape is essentially spherical. We can expect the gas to tend to escape along the direction of the minor axis of the density distribution. In the two cases examined, the difference of course is that we have the jet pushing along a defined direction which can be parallel or perpendicular to the minor axis. Therefore, at later times, we can expect different morphologies. Indeed, when the jet is oriented along the galaxy's major axis, the cocoon expands also laterally at relatively large speed due to the faster decrease in the external density in this direction. Furthermore, the distribution of the external gas causes a collimation of the outflow. This collimation is maintained also in the later stages of the evolution and produces an ordered outflow along the galaxy's minor axis and the overall morphology of the radio source is typical of the X-shaped sources. Conversely, when the jet is parallel the minor axis, a classical double radio source forms.

We conclude that the development of wings arises only when the jet is aligned with the major axis of the galaxy, as in this situation the lateral expansion of the radio source is favoured over the longitudinal expansion.

This result finds immediate confirmation in the observations: winged radio-source show a clear alignment between the principal radio and host axis (see Fig. 6), an effect which is not shared by the control sample. We conclude that wings naturally form in highly elliptical galaxies when the major radio axis form a small angle with the host galaxy's major axis.

\section{Discussion}

The results presented in the previous sections show that the origin of X-shaped radio-galaxies is a natural consequence of the expansion of the cocoon formed by the radio-source in a stratified medium with an elliptical distribution. This situation is reminiscent of the scenario envisaged by Blandford \& Rees (1974) in their twin-exhaust model: we have a bubble of hot and light gas inside an elliptical distribution of density and pressure. The bubble expands laterally (with respect to the jet axis) at a high rate due to the faster decrease in the external density in this direction with respect to the jet's direction. The external gas plays a second crucial role as it also collimates the cocoon expansion forcing the growth of well defined radio wings. Note that actually in our simulation, given the adopted cylindrical symmetry, a circular equatorial outflow is produced, that suffers a strong dilution. However, in a three-dimensional case, it is likely that the gas will escape in one dimension only, along the galaxy's minor axis, therefore maintaining a stronger thrust with respect to the outflow appearing in our results. For this reason we expect the cocoon lateral expansion to be even more prominent that suggested by the simulations.

The wings expansion progresses until the jet exits the central region of high density. At this stage the head of the radiosource advances at a substantially higher speed than in the earlier phases; the material backflowing towards the nucleus is not able to reach back the central cavity and stops to provide pressure support for the lateral expansion. Clearly when and how this will affect the radio-source morphology can only be assessed with more detailed three-dimensional simulations and with better information on the external gas distribution.

It is important to stress that the physical mechanism that we propose as the origin of X-shaped radio-sources is different from those suggested in the past, not only from those suggesting jet reorientation discussed below, but also from those in which the wings are formed by other hydrodinamical effects. In particular Worrall et al. (1995) and Leahy \& Williams (1984) emphasized the importance of buoyancy forces acting on the backflow. While buoyancy can also be important in our framework, we suggest that the driving mechanism at the origin of the radio-wings is the presence of an overpressured cocoon. In fact, as noted above, it might be problematic to channel the backflowing plasma in the wings once the radio-source is fully developed, as these models predict.

The results presented here, i.e. the connection between the properties of the host galaxy and the presence of a X-shaped radio morphology, appear to represent a significant problem for the competing model based on jet re-orientation. In fact it remains to be explained why the re-orientation should occur in a small fraction of radio-galaxies and preferentially in objects in which the jet is aligned with the galaxy minor axis. A second observational evidence, which is difficult to account in the framework of jet realignment, is the lack of winged FR I. In our scenario this is readily interpreted as FR I do not form the overpressured cocoons that are one of the necessary ingredient to lead to the formation of the wings.

Constraints on the wings origin may come from the radio spectral index mapping of these radio-sources as this can be 

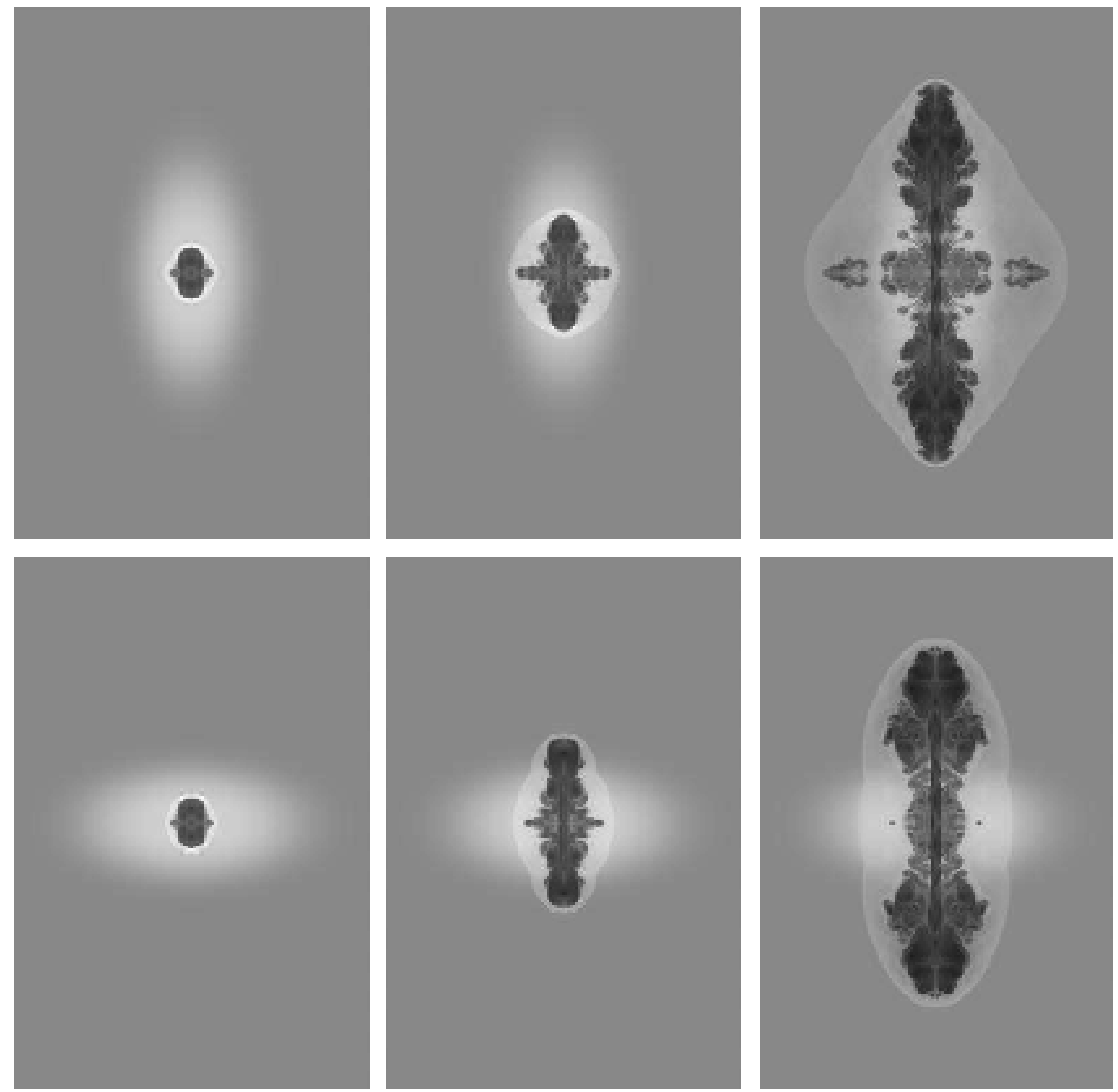

Fig. 5. Results of numerical simulations: we show density images representing the evolution of the radio-source in the stratified medium. The top panels refer to the case when the jet is oriented parallel to the major axis of the gas distribution, while the lower panels to the case when the jet is oriented parallel to the minor axis.

related to the age of the different radio-structures. In the context of jet re-orientation, wings represent aged relic of previous activity and the similarity of the spectral index on the main lobes and on the wings (Dennett-Thorpe et al. 2002) can be used to set limits on the realignment timescale. Conversely, in our scenario the radio wings are formed by jet material that flowed back to the central regions of the radio source and is then channeled away by the pressure excess of the cocoon. During this process the relativistic plasma experiences radiative losses (via synchrotron radiation and up-scattering of cosmic background photons) but, on the other hand, wings are continuously replenished with newly injected plasma and are tracers of an active outflow. In this situation significant reacceleration can occur due to shocks or turbulence and no simple prediction on the spectral index behaviour can be obtained.

Our results can also be tested by looking for counter examples, i.e. a source with the proper geometrical setup but without wings. For example, we found that wings are only present in galaxies with high ellipticity $(\epsilon>0.17)$. However, there are 6 objects in the control sample with isophotes ellipticity larger than this values, but without radio wings. It is worth discussing these objects individually. In two cases (3C 319 and $3 \mathrm{C} 456)$ the jets are almost aligned with the galaxy minor axis (within $20^{\circ}$ and $30^{\circ}$ respectively) and, as shown above, 


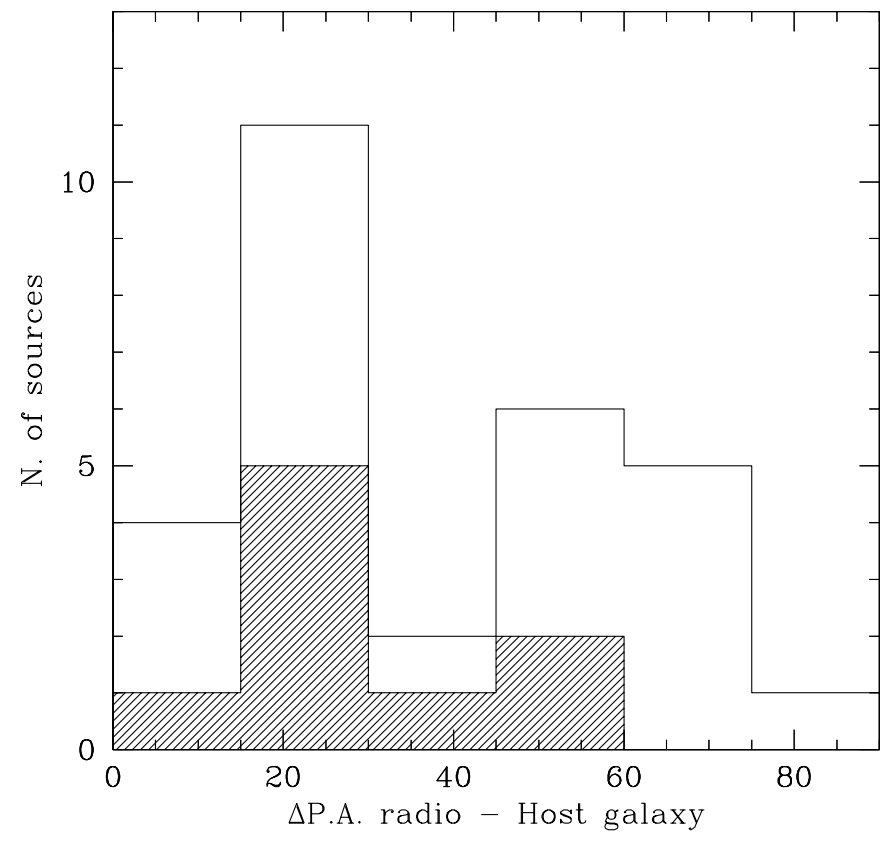

Fig. 6. Relative orientation of the major axis of the host galaxy and of the primary axis of radio emission.

in this geometrical configuration we do not expect the formation of wings; 3C 357 shows a centro-symmetric lobe distortion (see Harvanek \& Hardcastle 1998) although not well developed as in the X-shaped sources; 3C 326 is a giant radiogalaxy (its size exceeds $2 \mathrm{Mpc}$, Willis \& Strom 1978) and as such it probably evolved to a stage in which it is not influenced by the gas distribution on galactic scale; for the remaining two objects, 3C 327 and 3C 452, there are simply no high quality images in the literature to be used to explore the morphology of their lobes. Therefore we could not find radiosources representing strong counter examples conflicting with our model.

In our scenario two geometrical requirements must be fulfilled to produce a winged radio-source. This is particularly important when one wants to assess the relevance of this class. In fact, $\mathrm{X}$-shaped morphology are observed in a relatively small fraction (7\%) of FR II radio-sources (Leahy \& Parma 1992). This value suggests that they represent an intriguing but somewhat limited class. On the contrary, we argue that they develop essentially in all situations that fulfill the proper geometrical requirements: i) jets must form a large angle with the galaxy minor axis and ii) wings can occur only in galaxy of large ellipticity. Furthermore, projection can hide genuine X-shaped sources when e.g. the wings project onto the lobes, or either the wings or the jets are aligned with the line of sight. Finally, our definition of X-shaped radio-source is quite strict and several additional objects (as e.g. those cited in Sect. 2) might qualify if a looser definition is adopted. Note also that in several cases radio maps adequate to show the presence of diffuse wings are simply not available. Thus we argue that X-shaped radio-sources should not be viewed as peculiar objects but as the natural manifestation of the growth of the cocoon in a flattened distribution of external gas.

Finally, it appears that the proposed model can find application also in interpreting the evolution of other classes of radiosources. For example, the rapid lateral expansion due to gas stratification has been invoked by Capetti et al. (1999) to explain the dynamical properties of emission line cocoon formed by the radio-jets in the Seyfert galaxy Mrk 3. Furthermore, a flattened distribution of external gas can be produced by, e.g., the presence of a companion galaxy in a common envelope. In this case the external gas will have a symmetric distribution on galactic scales and an elliptical shape only on larger scales. Its asymmetry will start to affect the cocoon only when the radio-source has expanded to a size similar to the separation of the galaxies forming the double system; this might explain the Z-shaped morphology of the radio-source NGC 326 whose host is one component of a galaxies pair (see Murgia et al. 2001).

\section{Summary and conclusions}

We presented evidence that the orientation of the secondary axis of radio emission in $\mathrm{X}$-shaped radio-sources is closely linked to the orientation of the host galaxy. More specifically, considering the effects of projection, the radio wings appear to be parallel to the galaxy minor axis. This relationship suggests a causal connection between the galaxy property and the origin of the $\mathrm{X}$-shaped radio morphology. Such a connection is strengthened noting that $\mathrm{X}$-shaped radio sources are found only in galaxy of high ellipticity. We then argue that X-shaped radio-sources naturally form when a jet propagates in a nonspherical gas distribution. In this case the cocoon expansion along the direction of maximum pressure gradient (the galaxy minor axis) can occur at a similar (or higher) rate than along the radio source main axis. The situation is reminiscent of the twin-exhaust scenario where a bubble of hot and light gas inside an elliptical distribution of density and pressure expands laterally at a high rate producing wings of radio emission. Hydrodynamical simulations of the radio-source evolution in this situation support this scenario.

From the point of numerical simulations, a three dimensional analysis is clearly needed to investigate in more detail the radio source behaviour, but it might be envisaged that with more realistic simulations it might be possible to constraints the jet's properties based on the wings formation. In particular it will be important to perform a thorough exploration of the jet's parameters space. In fact, it is clear that the effects of the asymmetric gas distribution will affect more jets of lower kinetic power: at higher power the crossing time of the galaxy's central regions will be shorter and this will in turn shorten the phase during which the cocoon can expand laterally. We thus expect that only jets in a limited region of the parameters space might produced winged radio-sources.

Finally, a very valuable information will come from mapping of the external gas from high resolution X-ray images such as those that can now be obtained with the new generation of $\mathrm{X}$-ray satellites such as Chandra of Newton-XMM.

Acknowledgements. The authors acknowledge the Italian MIUR for financial support, grant No. 2001-028773. The numerical calculations have been performed at CINECA in BOlogna, Italy, thanks to the support of INAF. This research has made use of the NASA/IPAC 
Extragalactic Database (NED) which is operated by the Jet Propulsion Laboratory, California Institute of Technology, under contract with the National Aeronautics and Space Administration.

\section{References}

Blandford, R. D., \& Rees, M. J. 1974, MNRAS, 169, 395

Capetti, A., Axon, D. J., Macchetto, F. D., Marconi, A., \& Winge, C. 1999, ApJ, 516, 187

Capetti, A., Chiaberge, M., \& Celotti, A. 2002, in preparation Colella, P., \& Woodward, P. R. 1984, J. Comp. Phys., 54, 174

Dennett-Thorpe, J., Scheuer, P. A. G., Laing, R. A., et al. 2002, MNRAS, 330, 609

Ekers, R. D, Fanti, R., Lari, C., \& Parma, P. 1978, Nature, 276, 588

Fanaroff, B. L., \& Riley, J. M. 1974, MNRAS, 167, 31
Harvanek, M., \& Hardcastle, M. J. 1998, ApJS, 119, 25

Klein, U., Mack, K.-H., Gregorini, L., \& Parma, P. 1995, A\&A, 303, 427

Leahy, J. P., \& Williams, A. G. 1984, MNRAS, 210, 929

Leahy, J. P., \& Parma, P. 1992, in Extragalactic radio sources: From beams to jets, ed. J. Roland, H. Sol, \& G. Pellettier (C.U.P., Cambridge), 307

Murgia, M., Parma, P., de Ruiter, H. R., et al. 2001, A\&A, 380, 102

O’Dea, C. P., \& Owen, F. N. 1986, ApJ, 301, 841

Rees, M. J. 1978, Nature, 275, 516

Wirth, A., Smarr, L., \& Gallagher, J. S. 1982, AJ, 87, 602

Willis, A. G., \& Strom, R. G. 1978, A\&A, 62, 375

Worrall, D. M., Birkinshaw, M., \& Cameron, R. A. 1995, ApJ, 449, 93 